\title{
Review
}

Haiwei Dong*, Xiaohui Yang

\section{Research progress on the correlation between gestational vaginal microecology and vaginal infectious diseases}

DOI: $10.2478 / \mathrm{ii}-2018-0007$

Received January 28, 2018; accepted February 06, 2018; published online April 10, 2018

\begin{abstract}
The health status of the vaginal microenvironment, a complicated system, is an important indicator of female reproductive health. The vaginal flora is in a state of balance, and the microorganisms coexist and are interdependent to maintain the vaginal microecological balance, which is a kind of dynamic balance influenced by endogenous and exogenous factors. Vaginal infections are traditionally treated by killing microbes in the vagina. Given the extensive study of the internal vaginal environment, people have become gradually aware of the significance of maintaining the vaginal microecological balance rather than blindly using antimicrobial agents. The balance in the vaginal internal environment is disrupted during the gestation period as the secretion of progesterone increases. The imbalanced vaginal microecological environment may lead to vaginal infectious diseases. This article provides a review of the relationship between the vaginal microecology and infectious diseases during the gestation period.
\end{abstract}

Keywords: gestation period, vaginal microecology, vaginal infectious diseases, research progress

Vaginal infection during pregnancy significantly affects the health of the mother and baby. A pregnant woman with infection during pregnancy has a significantly higher probability of her baby being infected by a disease after birth than a pregnant woman who does not have vaginal infection. In addition, during childbirth, some cases of vaginal infection affect the health of the newborns. Therefore, studies have focused on the cause and prevention of vaginal infection during pregnancy. Research results show that vaginal microecology during pregnancy is related to vaginal infectious diseases. This result has been highly valued by clinicians in recent years $[1,2]$. This review summarizes the correlation between vaginal microecology and vaginal infection during the gestation period by reviewing recent clinical literature to improve the understanding of clinicians and perfect the treatment scheme for vaginal microecological-related diseases during pregnancy.

\section{Summary of vaginal microecology}

\subsection{Summary}

The vagina is a cavity that connects directly to the outside world and consists of a layer of microbes on its surface. Under normal conditions, Lactobacillus plays a major role in the vagina. Lactic acid and hydrogen peroxide produced by Lactobacillus not only inhibit the proliferation of endogenous pathogenic bacteria but also cease the foreign invasion of microorganisms. Therefore, maintaining the normal level of Lactobacillus in the vagina is of great significance because it can be used as an important indicator for measuring the intravaginal environment. 


\subsection{Bacterial flora distribution in the vagina}

The flora in the vagina consists of approximately 30 kinds of microbes, which are mainly distributed in the mucous membrane of the lateral wall in the vagina [3] and a few of them in the cervix also. Lactobacillus is the most common pathogen and plays a very important role in the vagina [4,5]. Lactobacillus can produce a large amount of lactic acid in the anaerobic respiration of glycogen to make the vaginal environment acidic, which helps to inhibit the propagation of bacteria. Lactobacillus inhibits exogenous pathogenic bacteria because of the following reasons: (1) it has strong absorbability to the mucous membrane of the vagina and thus can reduce the adsorption of other pathogenic bacteria to the vaginal mucosa and prevent the long-term existence of the pathogenic bacteria in the vagina; (2) it can produce hydrogen peroxide, which can inhibit the growth of pathogenic bacteria via disinfection and sterilization; and (3) it possesses a nutritive competitive effect that competes with other nutrients to inhibit the growth of pathogenic bacteria. Lactobacillus plays a leading role in the distribution and balance of the vaginal flora.

\subsection{Main factors affecting vaginal microecology}

Factors affecting intravaginal environmental balance include the following: $\mathrm{pH}$ of the vagina, changes in the flora caused by different physiological periods in women, secretion of sex hormones, sexual life, pregnancy or infertility, application of contraceptive devices (condom, vaginal rubber ring, and contraceptive diaphragm), pregnancy and childbirth, and other special physiological phenomena [6]. In addition, the personal life and sexual habits of women and social and economic factors have certain effects. The vaginal microenvironment is mainly evaluated in clinical settings by combining $\mathrm{pH}$ value and hydrogen peroxide in the vagina. If these indices are abnormal, then the vaginal microenvironment can be diagnosed to be damaged.

\section{Factors affecting the vaginal microecology during the gestation period}

\subsection{Endogenous factors}

Lactobacillus produced during the gestation period can significantly decrease the $\mathrm{pH}$ of the vagina. Hence, the vagina is strengthened to resist the invasion of foreign microorganisms. The $\mathrm{pH}$ of the vagina gradually increases with continuous promotion of pregnancy and is significantly increased compared with that in nonpregnancy. The increase in the $\mathrm{pH}$ value inevitably reduces the resistance of the vagina to external microorganisms and affects its microenvironment [7]. Mycoplasma and Chlamydia are common pathogens infecting the reproductive tract of women during pregnancy [8].

\subsection{Exogenous factors}

Local and systemic antibacterial agents affect the vaginal flora. Antibacterials can treat the vaginal infection and perform targeted killing of pathogenic bacteria to effectively prevent their proliferation. However, antibacterials affect other normal flora while killing the infectious bacteria, thereby disrupting the balance in the vaginal flora and increasing the probability of infection or the possibility of double infection [9]. Sex life is another exogenous factor. The $\mathrm{pH}$ of the vagina is always in an acidic state, whereas the $\mathrm{pH}$ of the semen and the mucus is alkaline; as such, during sex, the vaginal $\mathrm{pH}$ increases. Moreover, the vagina takes time to recover from acidity, and its resistance to external microorganisms 
is very low during the recovery. Therefore, frequent sex increases the probability of vaginal infection in women [10]. Vaginal irrigation is a popular method of cleaning the vagina with flushing fluid in foreign countries. Common flushing fluids include water, boric acid, and hydrogen peroxide. These fluids change the internal environment of the vagina, including its $\mathrm{pH}$, thereby changing the vaginal flora and increasing the incidence of bacterial infections in the vagina. Some studies have shown that vaginal irrigation leads to poor pregnancy outcomes, but other works report no adverse effect [11]. Therefore, a consensus has not been reached regarding this matter. However, avoidance of vaginal irrigation during pregnancy has become a common understanding.

\section{Genital infection diseases caused by vaginal microecology disorder during pregnancy and treatment}

\subsection{Vulvovaginal candidiasis (VVC)}

VVC results in vaginitis caused by the excessive growth of Candida. The incidence of VVC during the gestation period is very high, that is, twice of that during the non-gestation period [12]. This infection is retrograde, leading to the inflammation of the uterus and even abortion and preterm birth in severe cases. Thus, this infection must be given sufficient attention [13]. Appropriate supplementation of Lactobacillus in the body can effectively prevent the occurrence of vaginal candidiasis. The daily use of yogurt containing Lactobacillus can reduce the incidence of infection. Moreover, Lactobacillus inhibits VVC by positioning or competitive nutrients. Qi [10] discovered that the combined treatment of antifungal drugs and Lactobacillus can significantly improve the efficiency of the treatment and prevent recurrence of the disease.

\subsection{Bacterial vaginopathy (BV)}

BV refers to the reduction in the amount of hydrogen peroxide in the vagina during pregnancy, leading to reduced vaginal $\mathrm{pH}$ and inhibitory effect on foreign microbes; such a phenomenon causes an increase in the number of abnormal microbes, including bacteria, Mycoplasma, and anaerobes in the vagina. Patients with BV inflammation may experience adverse pregnancy outcomes, even miscarriages [14]. This infection is traditionally treated using metronidazole and other antibacterials, which have a certain effect and can kill bacteria effectively. With the continuous study of the internal environment of the vagina and the application of antimicrobial agents, reports show the increasing prominence of adverse reactions. People shift their attention to the treatment of BV by controlling the internal environment of the vagina. Hantoushzadeh et al. [15] discovered that the treatment effect of antibacterials is equal to that of Lactobacillus. However, Lactobacillus can effectively recover the balance of the internal environment of the vagina and treat vaginal infection caused by bacteria without any adverse reaction. This finding must be further evaluated through clinical studies.

\subsection{Aerobic vaginitis (AV) caused by aerobic bacteria}

$\mathrm{AV}$ refers to the imbalance of flora in the body caused by mass reproduction of Staphylococcus and Enterococcus in the vagina. The internal environment of the vagina during pregnancy changes, resulting in a reduced number of Lactobacillus and increased number of aerobic bacteria; this phenomenon leads to vaginitis. No ideal treatment method has been established for vaginitis caused by aerobic bacteria during pregnancy. In $\mathrm{AV}$, hydrogen peroxide from Lactobacillus in the vagina is largely lost. The supplementation of Lactobacillus exhibits a certain treatment effect. 


\subsection{Trichomonad vaginitis (TV)}

TV refers to a type of vaginitis caused by trichomonads in the vagina. The incidence of TV is high during the gestation period, that is, approximately $10 \%$ in pregnant women, which is higher than that in non-pregnant women. TV affects the health of women during pregnancy and the outcome of pregnancy. This infection is possibly related to poor pregnancy outcomes, such as preterm birth and premature rupture of membranes. Therefore, positive treatment must be conducted. Treatment of TV has changed from traditional antibacterials to exogenous Lactobacillus. The addition of exogenous Lactobacillus can effectively reduce the acidity of the vagina, thereby directly inhibiting Trichomonas. Exogenous application of Lactobacillus for treatment of TV exhibits high cure rate and high efficiency.

\subsection{Mixed infection in the vagina}

VVC, anaerobic bacteria, and aerobic bacteria are the main pathogens causing vaginitis. Vaginitis is not caused by a single bacterium but by several bacteria [16]. Zhang et al. [17] and Zhao [18] reported that approximately $50.0 \%$ of vaginitis cases are caused by mixed infection. Yu [19] and Yu and Fan [20] studied 230 patients infected with vaginitis caused by aerobic bacteria; the results showed that 156 cases of vaginitis were purely caused by aerobic bacteria, and the other 74 cases were due to mixed infection. Yan [21] and Li et al. [22] studied 1099 patients with vaginitis, of which $44.7 \%$ women had mixed infection. The mixed and single infections of the female reproductive system are similar in terms of clinical manifestations, but their symptoms are sometimes atypical. Hence, the type of infection is difficult to determine based on symptoms. Patients with mixed infection experience stronger pain, higher acid value, and less Lactobacillus in the vagina than those with single infection. During a gynecologic examination of a woman with atypical secretions, the infection must be assessed if it is mixed or single. If the patient is diagnosed to experience mixed infection, then timely evaluation of the internal environment of vagina must be performed and imbalanced flora must be recovered to avoid the invasion of opportunistic pathogens.

\section{Significance of the evaluation of vaginal microecology during the gestation period}

Gestation period is a physiological period that most women experience. The number of Lactobacillus in the vagina decreases during childbirth, resulting in severe effects on the balance of flora in the vagina. If exogenous microbes invade the vagina during this period (for example, frequent sex life would aggravate flora imbalance, resulting in vaginal infection), then the evaluation of microecology can lead to early judgment of the infection to determine whether sex life can be restored. The infection in pregnant women may cause adverse pregnancy outcomes, including abortion, premature rupture of membranes, and preterm delivery. Therefore, vaginal infection in pregnant women must be given sufficient attention. The microecology of the vagina during the gestation period dynamically changes and is easily affected by environmental change. During this period, the acid value in the vagina is not high and thus insufficient to kill pathogenic microbes. The mass reproduction of pathogens can easily occur, leading to vaginal infection. The relationship between general gestational vaginitis and adverse pregnancy outcome is often insufficient to explain the problem. Hence, the microecological environment of the vagina during pregnancy must be comprehensively assessed.

\section{Clinical application of vagina microecological therapy}

The vaginal microecosystem is characterized by a balanced and interdependent state among the vaginal flora. Under normal conditions, such microecology in the vagina is in the balanced state. After being affected 
by external and internal stimuli, a series of changes occurs in the internal environment of the vagina, leading to vaginitis. Lactobacillus is the dominant flora in the vagina. Hydrogen peroxide produced by Lactobacillus kills pathogenic microbes. Hence, Lactobacillus plays a lead role in the internal environment of the vagina. In this regard, the internal environment of the vagina must be adjusted by increasing the abundance of Lactobacillus and other dominant flora for treatment of various infectious diseases caused by imbalanced flora. Zhao treated bacterial vaginosis through different methods, such as increasing Lactobacillus in the vagina and using traditional antibacterials and observing different treatment effects. Compared with the treatment of increasing the dominant bacteria, the treatment method using pure antibacterials can kill pathogenic bacteria within a shorter period of time and can easily cause flora imbalance. Vaginitis can easily relapse. Simple treatment using dominant bacteria can restore the flora imbalance in the vagina. However, during the reproductive period of pathogens, simple addition of dominant bacteria cannot timely discharge pathogenic bacteria. Therefore, developing a more rapid and safe treatment is the key to curing vaginal diseases. The combined treatment of increasing the dominant bacteria and using antibacterials exhibits good clinical effects against vaginitis. In the early stage, antibacterials are used to kill pathogens on time to effectively inhibit their constant reproduction. The number of dominant bacteria increases timely restoration of the internal environment of the vagina and the imbalanced state of the flora. Hence, vaginal microecology plays a very important role in the treatment of vaginal infectious diseases.

Acknowledgments: None.

Conflict of interest: The author states no conflicts of interest.

Authors' contributions: $\mathrm{H}$ Dong made the literature analysis and wrote, discussed, and revised the manuscript of this review. X Yang critically analyzed and corrected the manuscript. All authors read and approved the final manuscript.

\section{References}

[1] Xiao B.B., Zhang D., Liao Q.P. Liu Z.H., Microecological evaluation of vaginal flora in gestation period, Chin. J. Clin. Obstetrics Gynecol., 2007, 8(6), 412-449.

[2] Xiao C.Y., Zhang H.W., Analysis on the composition of 2066 cases with vagina microecological imbalance, Int. J. Obstetrics Gynecol., 2012, 39(2), 191-193.

[3] Song J.H., Zheng J.J., Identification and analysis of vaginal lactobacillus in patients with bacterial vaginosis and healthy women in nationality of Pastoral area, Chin. J. Obstetrics Gynecol., 2011, 46(1), 41-44.

[4] Xiao B.B., Liao Q.P., Analysis on diversity of vaginal microbiota in healthy Chinese women by using DNA fingerprinting, J. Peking Univ. Med. Ed., 2012, 44(2), 281-287.

[5] Zhang D., A study of vaginal flora, J. Pract. Obstetrics Gynecol., 2010, 26(2), 89-90.

[6] Zhou Y., Liu Q.R., Analysis on reproductive tract infections of 4823 early pregnancy women, Lab. Med. Clin., 2012, 9(7), 822-823.

[7] Franchini M., Cianferoni L., Lippi G., Calonaci F., Calzolari S., Mazzini M., et al., Tubal sterilization by laparoscopy or hysteroscopy: which is the most cost effective procedure, Fertil. Steril., 2009, 91(4), 1499-1502.

[8] Huang C.Y., Wang R., Yao L., Analysis of lower genital tract infection during gestational period, Chin. Matern. Child Health Care, 2010, 25(28), 4101-4104.

[9] Zhang Y.M., Li Y.L., Wang L.N., Han Q., Wang H., Microecological evaluation of vaginal and cervical microflora in gestational period, China J. Emerg. Resusc. Disaster Med., 2009, 4(6), 384.

[10] Qi Y.Y., Clinical observation of amphotericin B vagina effervescent tablet combined with d'amphotericin in the treatment of vulvovaginal candidiasis in pregnancy, Clin. J. Med. Off., 2011, 39(2), 372.

[11] Derbent A.U., Ulukanlıgil M., Keskin E.A., Soylu G., Kafalı H. Does vaginal irrigation with saline solution in women with infectious vaginitis contribute to the clinical and microbiological results of antibiotic therapy? Gynecol. Obstet. Invest., 2012, 73(3), 195-200.

[12] Abad C.L., Safdar N., The role of lactobacillus probiotics in the treatment or prevention of urogenital infections-a systematic review, J. Chemother., 2009, 21(3), 243-252.

[13] Xue F.X., Progress in diagnosis and treatment of aerobic bacterial vaginitis, J. Pract. Obstet. Gynecol., 2010, 26(2), 84-85. 
[14] Fan A.P., Xue F.X., Analysis on clinic characteristics of aerobic bacterial vaginitis and mixed infection, Chin. J. Obstetrics Gynecol., 2010, 45(12), 904-908.

[15] Hantoushzadeh S., Sheikh M., Javadian P., Shariat M, Amini E., Abdollahi A., et al. Elevated vaginal pH in the absence of current vaginal infection, still a challenging obstetrical problem. J. Matern. Fetal Neonatal Med., 2014, 27(6), 582-587.

[16] Dong C.F., Zhang G.J., Wang Y.Q., The relationship between nongonococcal cervicitis and preterm birth as well as its effect on perinatal infant, Chin. Matern. Child Health Care, 2009, 24(4), 495-496.

[17] Zhang D.K., Qin C.R., Li X.Y., Shi C.J., Hu B., Cheng G., et al., A study on the microorganism of low genitalia infection among Guangzhou females, Chin. J. Microecol., 2005, 27(17), 417-418.

[18] Zhao G.L., The application of Lactobacillus vaginal capsule in the treatment of bacterial vaginosis, Chin. J. Mod. Drug Appl., 2011, 5(3), 109-120.

[19] Yu F.Z., Clinical characteristics of 230 cases of aerobic bacterial vaginitis (AV) and its mixed infection in outpatient clinic, Health Required, 2012, 11(3), 22-24.

[20] Yu Y.X., Fan X.J., Analysis of 40 cases of bacterial vaginosis treated with metronidazole vaginal effervescent tablets combined with diamphotericin, Contemp. Med., 2010, 6(4), 135-136.

[21] Yan X., Clinical observation of ornidazole combined with Yanhua in the treatment of bacterial vaginosis, Chin. Matern. Child Health Care, 2011, 27(26), 2231-2232.

[22] Li D.L., Ju J.B., Shi Q.X., Li G.M., Shi J.L., Study on the relationship between vaginal microbes and age, pregnancy, vaginal diseases, J. Dermatol. Venereol., 2012, 34(4), 234-236. 